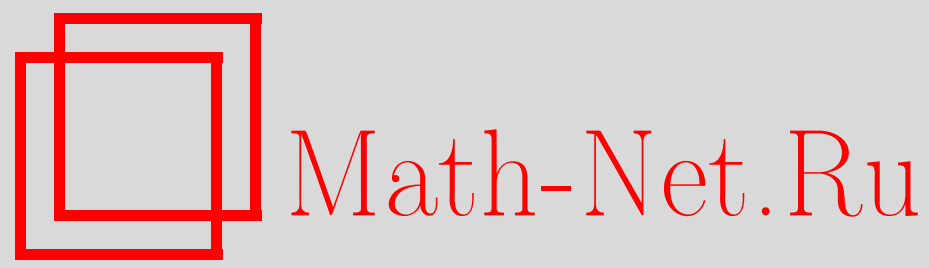

Ю. М. Мовсисян, Сверхтождества в многообразии решеток, Матем. заметки, 1996, том 59, выпуск $6,944-946$

DOI: https://doi.org/10.4213/mzm1796

Использование Общероссийского математического портала MathNet.Ru подразумевает, что вы прочитали и согласны с пользовательским соглашением

http://www . mathnet.ru/rus/agreement

Параметры загрузки:

IP : 3.85 .73 .92

26 апреля 2023 г., 10:21:40

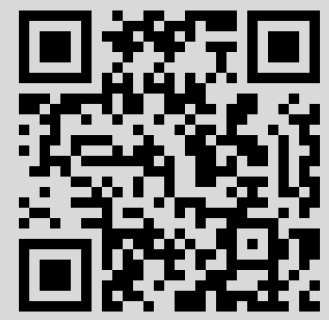




\section{СВЕРХТОЖДЕСТВА В МНОГООБРАЗИИ РЕШЕТОК}

\section{Ю.М. Мовсисян}

Сверхтождество (или $\forall(\forall)$ - тождество) - формула из языка второго порядка (ступени) [1], [2] вида

$$
\forall X_{1}, \ldots, X_{m} \quad \forall x_{1}, \ldots, x_{n} \quad\left(w_{1}=w_{2}\right),
$$

где $X_{1}, \ldots, X_{m}$ - функциональные переменные, а $x_{1}, \ldots, x_{n}$ - предметные переменные в термах (словах) $w_{1}, w_{2}$. Число $m$ называется функциональным рангом, а число $n$ - предметным рангом сверхтождества.

Сверхтождество кратко записывается без кванторной приставки: $w_{1}=w_{2}$. Выполнимость (истинность) этих формул второго порядка в алгебрах понимается с ограниченными (специализированными) функциональньми кванторами: в алгебре $\mathfrak{A}=\langle Q ; \Sigma\rangle$ выполняется сверхтождество

$$
w_{1}=w_{2},
$$

если равенство $(*)$ справедливо, когда в нем каждая предметная переменная и каждая функциональная переменная заменяются соответственно любым элементом из $Q$ и любой операцией, соответствующей арности из $\Sigma$; предполагается возможность такой замены, т.е.

$$
\left\{\left|X_{1}\right|, \ldots,\left|X_{m}\right|\right\} \subseteq\{|A| \mid A \in \Sigma\}=T_{\mathfrak{A}},
$$

где $|S|$ - арность $S$.

Сверхтождество $w_{1}=w_{2}$ называется сверхтождеством алгебры $\mathfrak{A}$ (многообразия $V$ ), если оно выполняется в алгебре $\mathfrak{A}$ (в любой алгебре $\mathfrak{A} \in V)$.

Алгебра $\mathfrak{A}=\langle Q ; \Sigma\rangle$ называется алгеброй с арифметическим типом $T \subseteq \mathbb{N}$ или $T$-алгеброй, если $T=T_{\mathfrak{A}}$. Сверхтождество называется $T$-сверхтождеством, если арность каждой его функциональной переменной содержится в $T$.

Указанная семантика согласована с гомоморфизмами $T$-алгебр [3], [4], определяемых как пары $(\varphi, \widetilde{\psi})$ отображений с условием

$$
\varphi A\left(x_{1}, \ldots, x_{n}\right)=[\widetilde{\psi}(A)]\left(\varphi x_{1}, \ldots, \varphi x_{m}\right) .
$$

$T$-сверхтождество $w_{1}=w_{2}$ называется следствием системы $T$-сверхтождеств $\mathscr{L}$, если всякий раз, когда любое сверхтождество из $\mathscr{L}$ выполняется в одной и той же $T$-алгебре, то и $T$-сверхтождество $w_{1}=w_{2}$ выполняется в ней, т.е. для любой $T$-алгебры $\mathfrak{A}$

$$
\mathfrak{A} \models \mathscr{L} \rightarrow \mathfrak{A} \models\left(w_{1}=w_{2}\right)
$$

(запись $\mathfrak{A}=\mathscr{L}$ означает выполнимость любого сверхтождества из $\mathscr{L}$ в алгебре $\mathfrak{A}$ ).

Перейдем к понятию формальног следствия системы $T$-сверхтождеств $\mathscr{L}$.

(i) Любое $T$-сверхтождество вида $w=w$ и любое $T$-сверхтождество из $\mathscr{L}$ являются формальньми следствиями $\mathscr{L}$.

(ii) Если $T$-сверхтождество $w_{1}=w_{2}$ является формальньм следствием $\mathscr{L}$, то и $T$-сверхтождество $w_{2}=w_{1}$ является формальным следствием $\mathscr{L}$. 
(iii) Если $T$-сверхтождества $w_{1}=w_{2}$ и $w_{2}=w_{3}$ являются формальными следствиями $\mathscr{L}$, то и $T$-сверхтождество $w_{1}=w_{3}$ является формальным следствием $\mathscr{L}$.

(iv) Если $T$-сверхтождество $u_{i}=v_{i}$ является формальным следствием $\mathscr{L}$ для $i=1, \ldots, n$, тогда для любого $n$-арного функционального переменного $Y$ (где $n \in T$ ) $T$-сверхтождество

$$
Y\left(u_{1}, \ldots, u_{n}\right)=Y\left(v_{1}, \ldots, v_{n}\right)
$$

также является формальным следствием $\mathscr{L}$.

(v) Если $T$-сверхтождество $w_{1}=w_{2}$ является формальным следствием $\mathscr{L}$, то и $T$-сверхтождество $w_{1}^{\prime}=w_{2}^{\prime}$ является формальным следствием $\mathscr{L}$, причем $w_{1}^{\prime}$ и $w_{2}^{\prime}$ получаются соответственно из $w_{1}$ и $w_{2}$ заменой всех вхождений произвольного функционального переменного $X$ (предметного переменного $x$ ) на любое функциональное переменное $Z$ той же арности (соответственно на любое $T$-слово $\omega$ ).

$T$-сверхтождество $w_{1}=w_{2}$ называется формальным следствием системы $T$-сверхтождеств $\mathscr{L}$, если оно является формальным следствием $\mathscr{L}$ согласно (i)-(v).

Tеорема А (о полноте для сверхтождеств). T-сверхтождество $w_{1}=w_{2}$ является следствием системь $T$-сверхтождеств $\mathscr{L}$ тогда и только тогда, когда оно является формальным следствием $\mathscr{L}$ [3], [4].

Сверхтождество $w_{1}=w_{2}$ назьвается полиномиальным сверхтождеством алгебры $\mathfrak{A}$ (многообразия $V$ ), если оно выполняется в полиномиальной алгебре $P(\mathfrak{A})$ алгебры $\mathfrak{A}$ (для любой алгебры $\mathfrak{A} \in V$ ).

Сверхтождество $w_{1}=w_{2}$ называется полиномиальным следствием системы сверхтождеств $\mathscr{L}$, если для любой алгебры $\mathfrak{A}$

$$
P(\mathfrak{A}) \models \mathscr{L} \rightarrow P(\mathfrak{A}) \models\left(w_{1}=w_{2}\right),
$$

где $P(\mathfrak{A})$ - полиномиальная алгебра алгебры $\mathfrak{A}$. Перейдем к понятию формалного полиномиального следствия системы $\mathbb{N}$-сверхтождеств $\mathscr{L}$.

(i') Совпадает с (i) при $T=\mathbb{N}$.

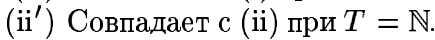

(iii') Совпадает с (iii) при $T=\mathbb{N}$.

(iv') Совпадает с (iv) при $T=\mathbb{N}$

$\left(\mathrm{v}^{\prime}\right)$ Совпадает с (v) при $T=\mathbb{N}$.

$\left(\mathrm{vi}^{\prime}\right)$ Если $\mathbb{N}$-сверхтождество $w_{1}=w_{2}$ является формальным полиномиальным следствием $\mathscr{L}$, то и $\mathbb{N}$ сверхтождество $w_{1}^{\prime \prime}=w_{2}^{\prime \prime}$ является формальным полиномиальным следствием $\mathscr{L}$, причем $w_{1}^{\prime \prime}$ и $w_{2}^{\prime \prime}$ получаются соответственно из $w_{1}$ и $w_{2}$ заменой всех вхождений произвольного функционального переменного $X$ на произвольный терм той же арности.

$\mathbb{N}$-сверхтождество $w_{1}=w_{2}$ назьвается формальным полиномиальным следствием системы $\mathbb{N}$-сверхтождеств $\mathscr{L}$, если оно является формальным полиномиальным следствием $\mathscr{L}$ согласно (i')-(vi'). 
ТЕОРЕма В (о полноте для полиномиальных сверхтождеств). $\mathbb{N}$-сверхтохсдество $w_{1}=w_{2}$ является полиномиальным следствием системы $\mathbb{N}$-сверхтожсдетв $\mathscr{L}$ тогда и только тогда, когда оно является формальным полиномиальным следствием $\mathscr{L}$.

Для характеризации сверхтождеств в многообразиях решеток, модулярных решеток и дистрибутивных решеток рассмотрим следуюшие сверхтождества:

$$
\begin{gathered}
X(x, x)=x \\
X(x, y)=X(y, x) \\
X(x, X(y, z))=X(X(x, y), z), \\
X(Y(X(x, y), z), Y(y, z))=Y(X(x, y), z), \\
X(Y(x, X(y, z)), Y(y, z))=Y(X(x, Y(y, z)), X(y, z)) \\
X(x, Y(y, z))=Y(X(x, y), X(x, z)), \\
X(u, X(x, X(y, v)))=X(u, X(y, X(x, v))), \\
X(Y(Y(y, x), z), Y(u, v))=X(Y(Y(y, x), z), X(Y(Y(u, x), v), Y(u, v))), \\
X(Y(Y(y, x), z), Y(u, x))=X(Y(Y(y, x), z), X(Y(Y(y, z), x), Y(u, x))) .
\end{gathered}
$$

ТЕорема 1. Любое сверхтохсдество многообразия решеток является следствием сверхтождеств (1)-(4).

ТЕОРема 2. Любое сверхтождество многообразия модулярных решеток является следствием сверхтохдеств (1)-(5).

ТЕорема 3. Любое сверхтождество многообразия дистрибутивных решеток является следствием сверхтождеств (1)-(3), (6).

ТЕОРема 4. Любое полиномиальное сверхтохдество с двумя бинарными функииональными переменными многообразия дистрибутивных решеток является полиномиальным следствием сверхтохсдетв (1), (3), (6)-(9).

Теорема 5. Существует такой конечный базис сверхтохдеств многообразия булевых алгебр, для сверхтохсдеств которого функииональные и предметние ранги не больше 3.

Характеризуются алгебры, удовлетворяющие сверхтождествам многообразий решеток, многообразия булевых алгебр.

Ереванский государственный университет

\section{СПИСОК ЦИТИРОВАННОЙ ЛИТЕРАТУРЫ}

1. Чёрч А. Введение в математическую логику. Т. 1. М.: ИЛ, 1961. 2. Мальцев А. И. Алгебраические системы. М.: Наука, 1970. 3. Мовсисян Ю. М. Введение в теорию алгебр со сверхтождествами. Ереван: Изд-во ЕГУ, 1986. 4. Мовсислн Ю. М. Сверхтождества и сверхмногообразия в алгебрах. Ереван: Изд-во ЕГУ, 1990. 